\title{
Discovery of the most distant star-forming and quenched galaxies in the universe
}

\author{
Steven L. Finkelstein \\ The University of Texas at Austin, Austin, TX, 78712, USA \\ email: stevenf@astro.as .utexas.edu
}

\begin{abstract}
While the high-redshift component of the CANDELS survey was designed with the $z \sim 6-8$ era in mind, these data do probe the far-UV of galaxies at even higher redshift. A few studies have ventured this far out, and have published conflicting results - some continue to find significant star-formation, while others conclude there is a steep decline in this quantity. Here I report on a new search for $z=9-10$ galaxies, making significant use of the Spitzer/IRAC data in the CANDELS fields. We have discovered a larger number of galaxies in this epoch than previous works, implying the UV luminosity function, and thus the SFR density, may not evolve as steeply as previously thought. This implies that star-formation begins early in the universe. I will also report on a new study searching for the earliest quenched galaxies at $3<z<5$, which are not predicted by models, yet may exist if galaxies form very early, and thus can approach their quenching phase quicker.
\end{abstract}

Keywords. galaxies: formation — galaxies: high-redshift

\section{Introduction}

While the past decade, since the addition of the infrared-sensitive Wide Field Camera 3 (WFC3) to the Hubble Space Telescope (HST), has seen the solution to a number of pressing questions, more have since been opened. How rapidly does the process of the reionization of the intergalactic medium (IGM) end, and what is the dominant driver? When do the first galaxies turn on, and how rapidly do they build up? And, how fast do these early galaxies begin the process of shutting down their star formation, producing quiescent galaxies? In this proceeding, I summarize progress in these three areas.

\section{How Rapidly Does Reionization End?}

Typical models of reionization from earlier in this decade were able to show that highredshift galaxies were capable of completing reionization by $z \sim 6$ with a few assumptions, most importantly that all galaxies have ionizing photon escape fractions of $\sim 10-20 \%$ (Finkelstein et al. 2012; Robertson et al. 2013; Finkelstein et al. 2015; Robertson et al. 2015; Bouwens et al. 2015). However, such high escape fractions are rarely seen, and most studies of the average galaxy populations at $z \sim 2-4$ find upper limits which are much lower (<5\%; e.g., Siana et al. 2010; Grazian et al. 2017). Simulations frequently predict that is the lowest-mass galaxies which have the highest escape fractions (e.g., Paardekooper et al. 2015; Xu et al. 2016). This was recently explored by Finkelstein et al. (2019b), who found that if one assumes the escape fraction is halo-mass dependent (following Paardekooper et al. (2015)), one can still complete reionization "on time" by $z \sim 6$, but reionization starts earlier and proceeds more smoothly, due to the dependence on the faintest galaxies. These two models differ strongly at $z \sim 7-10$. The first model predicts an IGM which is $\sim 50 \%$ neutral and $z=7$, while the second model predicts $\sim 20 \%$ neutral. This is summarized in Figure 1. 


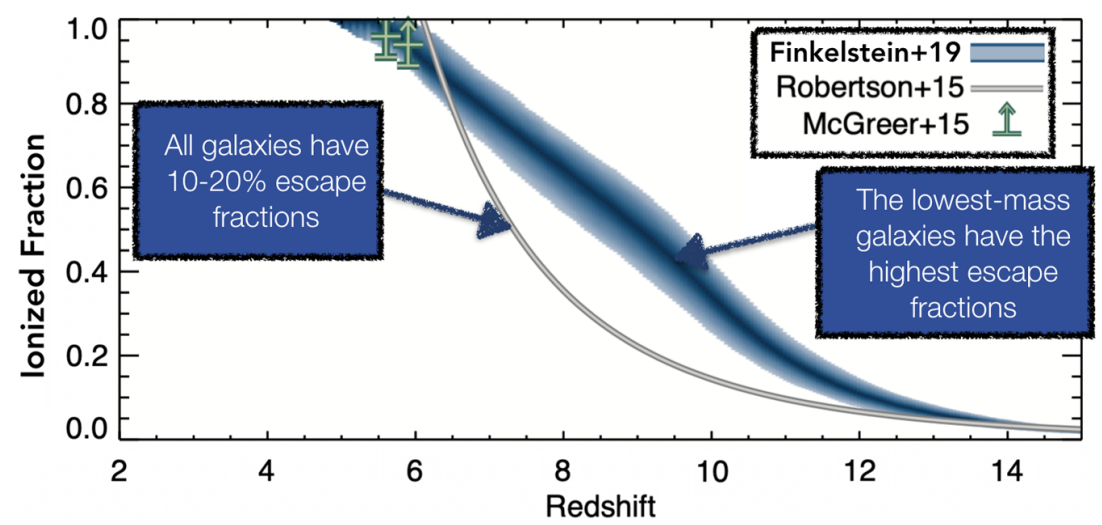

Figure 1. Adapted from Finkelstein et al. (2019b). The gray curve shows the reionization history predicted for a model where all galaxies contribute, with a constant escape fraction. The blue curve shows that from a model where only the faintest galaxies have significant escape fractions.

Additional observations of the neutral fraction in the epoch of reionization can help distinguish between these (and other) models. The most promising presently available method to do so is via Ly $\alpha$ emission. Ly $\alpha$ photons are resonantly scattered by neutral hydrogen, thus an increasing IGM neutral fraction should be traced by a decrease in detectable Lya emission (e.g., Miralda-Escudé \& Rees 1998; Malhotra \& Rhoads 2004). In spite of some detections (e.g., Shibuya et al. 2012; Finkelstein et al. 2013; Oesch et al. 2015; Zitrin et al. 2015; Stark et al. 2016; Hoag et al. 2017; Laporte et al. 2017; Larson et al. 2018), the majority of $z>7$ galaxies go undetected with spectroscopic followup. Based on these results, the most recent studies infer $\mathrm{X}_{H I}=60-90 \%$ at $\mathrm{z}=7-8$ (Mason et al. 2018, 2019; Hoag et al. 2019), due to just a few detections, and often none. However, converting observed spectra to constraints on the neutral fraction has lots of assumptions and potential for systematic uncertainties, including: incomplete spectral coverage of the full $P(z)$, copious telluric emission lines, uncertainty in spectroscopic depth of non-detections due to uncertain Ly $\alpha$ line width (and sometimes shallow depth), and sample contamination.

In the thesis work of newly minted Dr. Intae Jung (published in Jung et al. $(2018,2019)$ and Jung et al. (2019b)), we used data taken over 18 nights from Keck with DEIMOS and MOSFIRE to try to overcome some of these systematics, specifically:

- Depth: Our MOSFIRE integrations range from 5-20 hours.

- Sample selection: Significant effort to improve the photo-z's (and minimize sample contamination) of the observed galaxies.

- Wavelength: 20 of our sources are covered by both DEIMOS and MOSFIRE.

Out of our full MOSFIRE sample of 72 galaxies in the GOODS-N field observed with exposure times greater than 5 hours, we found nine Ly $\alpha$ lines at $7.1<z<7.9$ detected at $\geqslant 4 \sigma$ significance (including five at $\geqslant 5 \sigma$ ). We used our observations to model the Ly $\alpha$ equivalent width (EW) distribution, accounting for all sources of incompleteness and uncertainty by implementing via MCMC (similar to Jung et al. (2018)). We constrain the characteristic scale-length of this distribution, predicting for a given value the number of lines we should detect at a given $\mathrm{S} / \mathrm{N}$ in our data. While the scale length of Ly $\alpha$ emission is typically $\sim 70 \AA$ at $z<6$, we find $30 \pm 10 \AA$ at $z \sim 7.5$. We conclude that in contrast to previous results, Ly $\alpha$ is very detectable in this epoch. Our measured scale length implies a neutral fraction which is non-zero, but likely not as high as previously published. 


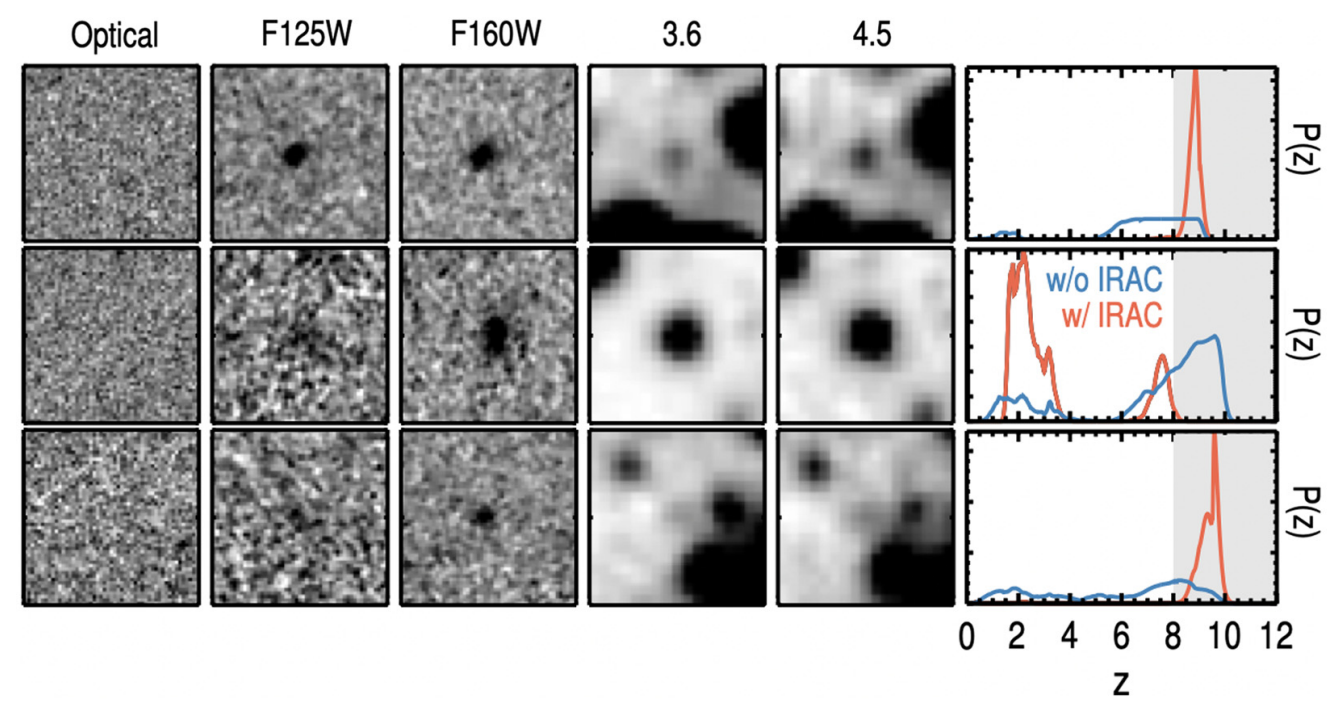

Figure 2. Adapted from Finkelstein et al. (2019c), in prep. Each row shows one object, with the right-hand panel showing the $P(z)$ without and with the inclusion of IRAC data. The first source is faint yet detectable in IRAC, with a red [3.6]- [4.5] color indicative of strong [O III] emission in the redder channel, consistent with $z \sim 8.5-9$. the second source is very bright in IRAC, showing a very red slope consistent with a low-redshift solution. The final source is not strongly detected in IRAC, again consistent with a high redshift nature.

\section{When do the First Massive Galaxies Form?}

While studies of the rest-ultraviolet (UV) luminosity function, and by extension the cosmic star-formation rate (SFR) density, agree nicely that there is smooth evolution from $z=4$ to $z=8$ (Finkelstein 2016), there is significant tension at higher redshifts. While studies by Coe et al. (2013) and McLeod et al. (2015) imply that the smooth evolution continues to $z \geqslant 9$, other studies by Bouwens et al. (2016) and Oesch et al. (2018) find an accelerated evolution downward. This discrepancy is understandable, as at $z>9$, galaxies are only robustly detected in a single $H S T$ filter (F160W), and are just barely detectable with this $2.4 \mathrm{~m}$ telescope. This is thus exceedingly difficult, and previous work pushing previous generations of HST instruments to their limits have shown that it is possible to underestimate the true abundance of such difficult-to-find galaxies (e.g. Bouwens et al. 2004). The hopefully imminent launch of the James Webb Space Telescope (JWST) will rapidly clear this up. The approved Cosmic Evolution Early Release Science (CEERS) Survey (Finkelstein et al. 2017) is designed to robustly measure the SFR density at $z \sim 10$, going $\sim$ two magnitudes deeper than CANDELS, and detecting $z \sim 10$ galaxies in 5-8 filters.

However, that is still a few years away. We can make progress now by using a telescope even smaller than Hubble - the Spitzer Space Telescope. While the sensitivity of Spitzer's IRAC camera is less than WFC3, deep surveys such as S-CANDELS (PI Fazio) and GREATS (PI Labbé) can detect rest-frame optical emission from galaxies at $z \sim 9-10$. As shown in Figure 2, the inclusion of Spitzer/IRAC photometry can significantly shrink the uncertainties on the photometric redshift probability distribution function, allowing the selection of a robust $z \sim 9-10$ galaxy candidate whose nature remained muddle with HST data only.

Inspired by this, we have embarked on a search for $z>8$ galaxies in the CANDELS fields including Spitzer/IRAC photometry in the photometric redshift selection, to try 


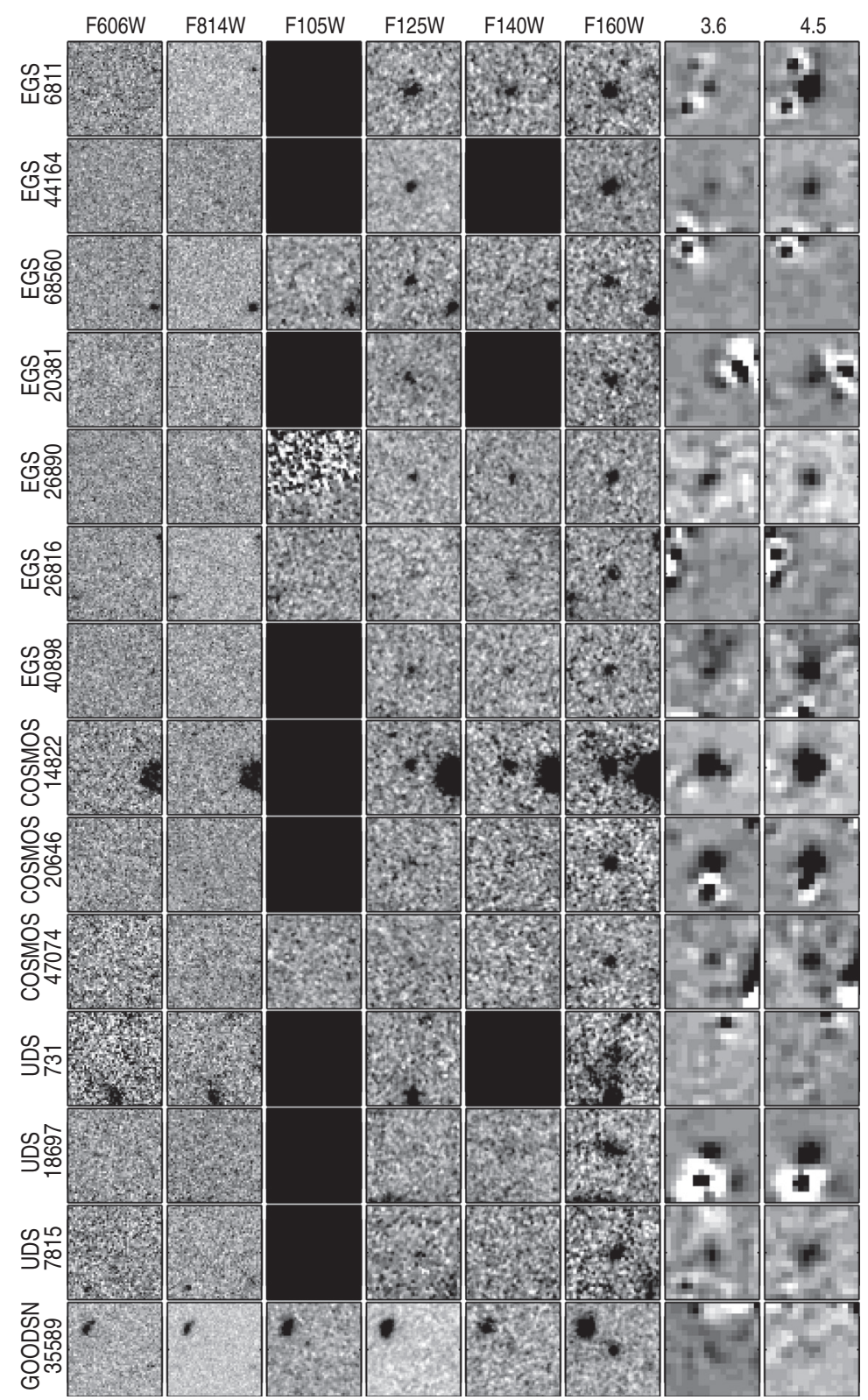

Figure 3. Adapted from Finkelstein et al. (2019c), in prep. Each row shows one candidate $z>8.5$ galaxy from the CANDELS fields, selected with both HST and Spitzer/IRAC imaging. Each column shows one filter, with the last two showing the two IRAC bands after subtraction of neighbors with TPHOT. Further inclusion of ground-based imaging show that the sources COSMOS 14822 and UDS 731 are likely low-redshift interlopers.

to better constrain the abundance of galaxies in this epoch. Over the five CANDELS fields, we have discovered 14 bright $H<26.5$ galaxies at $z>8.5$, larger than previously published samples. After an extensive vetting process including all available ground and space-based data (described in Finkelstein et al. (2019c), in prep), we find 12 of these sources remain very likely high-redshift candidates. This analysis is ongoing, but the presence of these 12 promising candidates results in a volume density of bright sources 


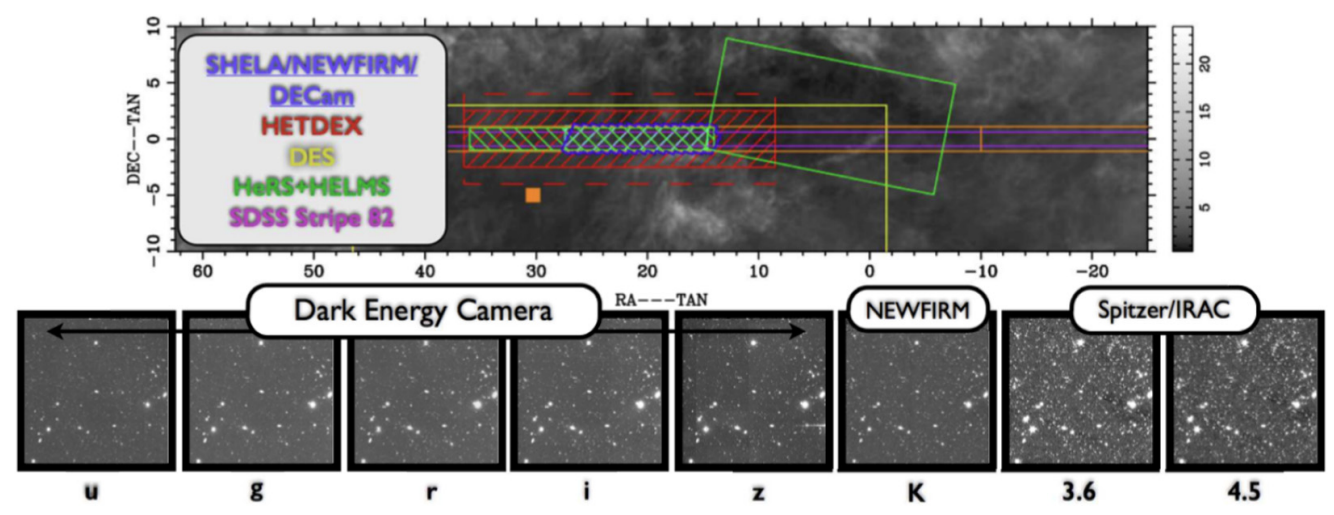

Figure 4. A schematic diagram of the $20 \mathrm{deg}^{2}$ SHELA field, highlighting the datasets available. We use these data, led by our new $K_{s}$-band imaging, to select candidate massive quiescent galaxies at $3<z<5$.

which is essentially unchanged from $z \sim 6$ to $z \sim 9$, presenting a challenge to models of early universe galaxy formation.

What could this mean? Maybe we are just bad at weeding out contaminants? That would be unsatisfying as we all work very hard at this, but we need spectroscopic confirmation. We are attempting to confirm the redshifts of these galaxies via Ly $\alpha$, with a MOSFIRE J-band program being led by Rebecca Larson, as well as [O III] $88 \mu \mathrm{m}$, with an ALMA program led by Takuya Hashimoto. If we confirm these sources to be real, bright, distant galaxies must be better at making stars, possibly due to a steepening of the star-formation law at high gas densities (Yung et al. (2019) shows this is needed to match high-redshift rest-UV luminosity functions).

\section{How early do galaxies begin to shut down their star formation?}

If the universe is making bright and massive galaxies earlier than predicted, might it also produce massive quiescent galaxies early as well? Models currently predict that all massive galaxies should be star-forming at $z>3$ (e.g., Brennan et al. 2015). Observational studies in this epoch show that such quiescent galaxies do exist at $z>3$ (e.g., Glazebrook et al. 2017), but the fraction of massive galaxies which are quiescent has been observed to range from $\sim 10-50 \%$. This spread is largely driven by the very small volumes probed by many studies.

To make progress, we are using the $20 \mathrm{deg}^{2}$ Spitzer-HETDEX Exploratory Large Area (SHELA; Figure 4) survey to perform a search for massive quiescent galaxies at $3<z<5$. This field contains fairly deep CTIO/DECam optical (Wold et al. 2019) and Spitzer/IRAC (Papovich et al. 2016) imaging. To this we have added $K_{s}, A B=23$ imaging from our 93 night KPNO/NEWFIRM survey program (PI Finkelstein; Stevans et al. in prep).

Our $K_{s}$-selected catalog of all 1.6 million sources in this field is presented in Stevans et al., in prep. We selected a sample of $\sim 4000$ massive $\left(\log \left[M / M_{\odot}\right]>11\right)$ galaxies with photometric redshifts between $3<z<5$ using EAZY-py. Using the measured EAZY-py SFRs, we then classified galaxies as quiescent if they had specific SFRs $<10^{-11} \mathrm{yr}^{-1}$, finding $\sim 500$ such galaxies. While this selection is difficult due to the limited number of photometric bands in this field, we can make two main conclusions at this point. First, this number is decidedly non-zero, therefore all models which predict it should be zero (which is all models presently) need revision. Simply increasing feedback may not be enough, as that will simply delay the formation of such massive galaxies. Second, 


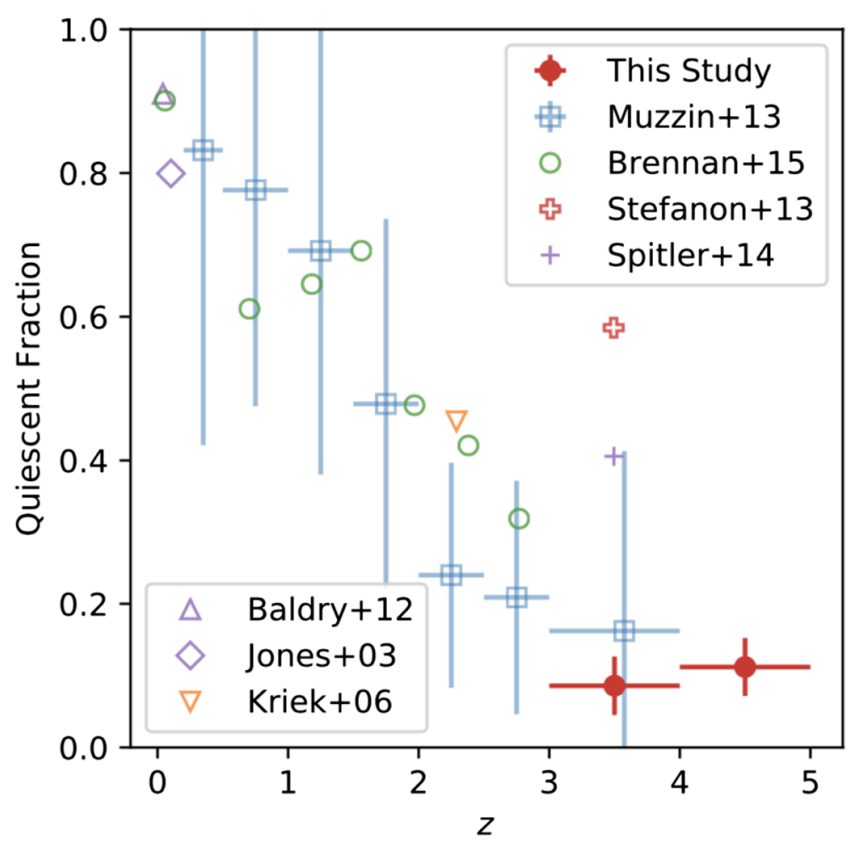

Figure 5. Adapted from Stevans et al. (2019). The quiescent fraction of massive galaxies (log $\left.\left[\mathrm{M} / \mathrm{M}_{\odot}\right]>11\right)$ as a function of redshift. The results from this study are shown in red, compared to a large number of studies from the literature. Our measure is at the lower end of previous observations, with significantly reduced uncertainties.

our measured quiescent fraction of $\sim 12 \pm 4 \%$ (Figure 5) is at the low-end of previous observational estimates, but with a much smaller uncertainty, and a negligible cosmic variance uncertainty. These observations tell us that whatever mechanism is responsible for quenching, it must be able to manifest rapidly, as these galaxies are quenched only 1.5 Gyr after the Big Bang.

\section{Lingering Questions}

In these proceedings, I have discussed new results on studies of reionization, and the formation and quenching of massive galaxies at high redshift. These results, while interesting, are far from conclusive. Here I discuss some lingering questions.

- What is the true reionization history? Our new Ly $\alpha$ observations imply a fairly ionized IGM at $z \sim 7.5$, although we have not yet estimated the neutral fraction from our dataset. If this is confirmed, how is it reconciled with other studies, which infer a much larger neutral fraction? Reionization cosmic variance could be to blame, though it could also highlight the difficulty of working at faint flux levels with ground-based nearIR spectroscopy. Some progress may be made with deeper, telluric-free, observations from $J W S T$, though the spectroscopic field-of-view is very small. Longer term, wide-field spectroscopic surveys with the Giant Magellan Telescope offer a promising future for Ly $\alpha$ based studies of reionization (more information on this can be found in the Astro2020 white paper by Finkelstein et al. (2019a)).

- Are the bright $z \sim 9-10$ candidates we've found real? Spectroscopic confirmation is needed to know for sure. ALMA observations of the [O III] $88 \mu \mathrm{m}$ line are likely the most efficient method in the pre- JWST era (Hashimoto et al. 2018). However, the majority of our sources are too far north for ALMA observations. NOEMA in principle can do this, but this requires NOEMA's Band 4, which has not yet been installed. Ly $\alpha$ remains a 
possibility, though this requires pushing current 10m-class telescopes to their limits, and is risky. Ultimately, rest-UV observations with JWST will likely be needed (or perhaps single-slit rest-optical observations with MIRI).

- If these candidates are real, why is the bright-end of the LF evolving so slowly? Is it due to a change in the physics regulating star-formation, or a reduction in dust attenuation? If the latter, once these galaxies hit dust-free, luminosity function evolution should continue, thus JWST observations pushing to $z>10$ should prove illuminating. Cosmic variance is also certainly playing a role, as can be seen by the variance in number of galaxies between our five fields $(7,2,2,1$ and 0 in the five CANDELS fields). Out to $z \sim 10$, WFIRST surveys will negate these uncertainties, improving these abundance estimates.

- What are the implications of massive quiescent galaxies forming at $z>3$ ? The presence must certainly inform the implementation of various forms of feedback in modern cosmological simulations. However, before we assume the model physics need revision, we need to make sure the observations are right. First, we need to test whether any of our candidate quiescent galaxies are dusty interlopers. This is easily done with minutes of ALMA observations, and we have a pilot program for 100 sources approved for Cycle 7 (PI Finkelstein). Once we have a clean sample of quiescent candidates, we can then pursue near-IR spectroscopic confirmation of the $4000 \AA$ break. Due to their bright magnitudes $\left(K_{A B} \sim 20-22\right)$, this should also be fairly quick, perhaps a few hours with Keck/MOSFIRE. Combining these results will provide us with a more reliable estimate of the quiescent fraction, which can then be used to constrain future simulations.

\section{References}

Bouwens, R. J., Illingworth, G. D., Oesch, P. A., et al. 2015, ApJ, 811, 140

Bouwens, R. J., Thompson, R. I., Illingworth, G. D., et al. 2004, ApJ, 616, L79

Bouwens, R. J., Oesch, P. A., Labbé, I., et al. 2016, ApJ, 830, 67

Brennan, R., Pandya, V., Somerville, R. S., et al. 2015, MNRAS, 451, 2933

Coe, D., Zitrin, A., Carrasco, M., et al. 2013, ApJ, 762, 32

Finkelstein, S., Dickinson, M., Ferguson, H., et al. 2017, The Cosmic Evolution Early Release Science (CEERS) Survey, JWST Proposal ID 1345. Cycle 0 Early Release Scienc

Finkelstein, S., Bradac, M., Casey, C., et al. 2019a, BAAS, 51, 221

Finkelstein, S. L. 2016, PASA, 33, e037

Finkelstein, S. L., Papovich, C., Ryan, R. E., et al. 2012, ApJ, 758, 93

Finkelstein, S. L., Papovich, C., Dickinson, M., et al. 2013, Nature, 502, 524

Finkelstein, S. L., Ryan, Jr., R. E., Papovich, C., et al. 2015, ApJ, 810, 71

Finkelstein, S. L., D'Aloisio, A., Paardekooper, J.-P., et al. 2019b, arXiv e-prints

Glazebrook, K., Schreiber, C., Labbé, I., et al. 2017, Nature, 544, 71

Grazian, A., Giallongo, E., Paris, D., et al. 2017, A\& $A$, 602, A18

Hashimoto, T., Laporte, N., Mawatari, K., et al. 2018, Nature, 557, 392

Hoag, A., Bradač, M., Trenti, M., et al. 2017, Nature Astronomy, 1, 0091

Hoag, A., Bradač, M., Huang, K., et al. 2019, ApJ, 878, 12

Jung, I., Finkelstein, S. L., Livermore, R. C., et al. 2018, ApJ, 864, 103

Jung, I., Finkelstein, S. L., Dickinson, M., et al. 2019, arXiv e-prints, arXiv:1901.05967

Laporte, N., Nakajima, K., Ellis, R. S., et al. 2017, ApJ, 851, 40

Larson, R. L., Finkelstein, S. L., Pirzkal, N., et al. 2018, ApJ, 858, 94

Malhotra, S. \& Rhoads, J. E. 2004, ApJL, 617, L5

Mason, C. A., Treu, T., Dijkstra, M., et al. 2018, ApJ, 856, 2

Mason, C. A., Fontana, A., Treu, T., et al. 2019, MNRAS, 485, 3947

McLeod, D. J., McLure, R. J., Dunlop, J. S., et al. 2015, MNRAS, 450, 3032

Miralda-Escudé, J. \& Rees, M. J. 1998, ApJ, 497, 21

Oesch, P. A., Bouwens, R. J., Illingworth, G. D., Labbé, I., \& Stefanon, M. 2018, ApJ, 855, 105 
Oesch, P. A., van Dokkum, P. G., Illingworth, G. D., et al. 2015, ApJL, 804, L30

Paardekooper, J.-P., Khochfar, S., \& Dalla Vecchia, C. 2015, MNRAS, 451, 2544

Papovich, C., Shipley, H. V., Mehrtens, N., et al. 2016, ApJS, 224, 28

Robertson, B. E., Ellis, R. S., Furlanetto, S. R., \& Dunlop, J. S. 2015, ApJL, 802, L19

Robertson, B. E., Furlanetto, S. R., Schneider, E., et al. 2013, ApJ, 768, 71

Shibuya, T., Kashikawa, N., Ota, K., et al. 2012, ApJ, 752, 114

Siana, B., Teplitz, H. I., Ferguson, H. C., et al. 2010, ApJ, 723, 241

Stark, D. P., Ellis, R. S., Charlot, S., et al. 2016, MNRAS

Wold, I. G. B., Kawinwanichakij, L., Stevans, M. L., et al. 2019, ApJS, 240, 5

Xu, H., Wise, J. H., Norman, M. L., Ahn, K., \& O'Shea, B. W. 2016, ApJ, 833, 84

Yung, L. Y. A., Somerville, R. S., Finkelstein, S. L., Popping, G., \& Davé, R. 2019, MNRAS, 483,2983

Zitrin, A., Labbé, I., Belli, S., et al. 2015, ApJL, 810, L12 